Chirurg 2013 $84: 1074$

DOI 10.1007/s00104-013-2652-3

Online publiziert: 14. November 2013

๑) Springer-Verlag Berlin Heidelberg 2013

F. Brink ·. Jähne

Klinik für Allgemein- und Viszeralchirurgie, Schwerpunkt für endokrine und onkologische Chirurgie,

Diakoniekrankenhaus Henriettenstiftung gGmbH, Hannover

\title{
Laparoskopische anteriore Hemifundoplikation vs. Nissen-Fundoplikation
}

\section{Langzeitergebnisse}

\section{Originalpublikation}

Broeders JA, Broeders EA, Watson DI et al (2013) Objective outcomes 14 years after laparoscopic anterior 180-degree partial versus Nissen fundoplication: results from a randomized trial. Ann Surg 258:233-239

\section{Hintergrund}

Die Resultate klinisch randomisierter Studien bezüglich der anterioren Hemifundoplikation (AHFP) suggerieren eine gleichwertige Kontrolle der Refluxsymptomatik bei geringeren Nebenwirkungen als bei der Fundoplikation nach Nissen. Objektive Ergebnisse einer späten Followup-Studie, die dies untermauern, wurden bis dato nicht veröffentlicht.

\section{Patienten und Methoden}

An der ursprünglichen randomisierten Studie zur AHFP vs. Nissen-Fundoplikation nahmen 103 Patienten teil. Im Rahmen des Follow-ups nach 14 Jahren wurden 18 Patienten (8 AHFP und 10 Nissen) einer hochauflösenden Ösophagusmanometrie und einer 24-Stunden-Impedanz$\mathrm{pH}$-Metrie unterzogen. Diese Gruppe sowie 59 weitere Patienten füllten einen standardisierten klinischen Fragebogen aus.

\section{Ergebnisse}

Beim Follow-up wiesen untersuchte und nichtuntersuchte Patienten ein vergleich- bares Symptomprofil auf. Die Untersuchungen ergaben, dass die totale ösophageale Säureexpositionszeit und die Anzahl der starken und schwachen Säurerefluxepisoden pro $24 \mathrm{~h}$ bei der AHFP höher waren als bei der Operation nach Nissen. Proximaler, mittlerer und distaler ösophagealer Reflux waren nach AHFP proportional erhöht, die mittlere Ausdehnung des Refluxes war bei beiden Methoden jedoch gleich. Ebenso war die Anzahl flüssiger und gemischter Refluxepisoden nach AHFP erhöht, begleitet von einem erhöhten klinischen Heartburn-Score. Keine Unterschiede zeigten sich in Gasreflux, gastrischem sowie supragastrischem Aufstoßen. Der mittlere Ruhe- und Entspannungsdruck des unteren Ösophagussphinkters war geringer nach AHFP, was sich in niedrigeren Dysphagie-Scores widerspiegelt. Die Patientenzufriedenheit war bei beiden Prozeduren gleich.

\section{Diskussion}

Die Fundoplikation nach Nissen ist aktuell die am häufigsten durchgeführte operative Methode zur Therapie von Refluxerkrankungen. Zuletzt publizierte Metaanlysen von Follow-up-Studien bis zu 5 Jahre nach $180^{\circ}$ - und $270^{\circ}$-Fundoplikation suggerieren eine gleichwertige Kontrolle der Refluxsymptomatik bei weniger Nebenwirkungen bezogen auf Dysphagie und gasbezogene Symptomatik.

Aus den oben genannten Ergebnissen dieser Studie ergibt sich zum einen eine weniger effektive Refluxkontrolle nach
AHFP, zum anderen besteht postoperativ weniger Dysphagie. Die Autoren sprechen daher von einem vergleichbaren klinischen Ergebnis nach beiden Operationsmethoden im Langzeit-Follow-up.

Durch die geringe Anzahl der objektiv untersuchten Patienten, die in Vergleich zu einem subjektiven Fragebogen gesetzt werden, ist die absolute Aussagekraft dieser Studie kritisch zu sehen, was den Autoren durchaus bewusst ist.

Langfristig scheint die Fundoplikation nach Nissen die Methode der Wahl zu sein, während bei älteren Patienten aufgrund der geringeren Nebenwirkungen (insbesondere Dysphagie) eine AHFP vorteilhaft erscheint. Letztendlich reicht die Datenlage jedoch nicht aus, hier eine eindeutige Empfehlung auszusprechen, sodass weitere Langzeitstudien mit einem größeren Patientenpool erforderlich sind.

\section{Korrespondenzadresse}

\section{F. Brink}

Klinik für Allgemein- und Viszeralchirurgie, Schwerpunkt für endokrine und onkologische Chirurgie, Diakoniekrankenhaus Henriettenstiftung $\mathrm{gGmbH}$, Marienstr. 72-90, 30171 Hannover Felix.Brink@ddh-gruppe.de

Interessenkonflikt. F. Brink und J. Jähne geben an, dass kein Interessenkonflikt besteht. 